\title{
Determining the effect of time dependent and time independent factors on pneumonia of children under five in North west Ethiopia
}

\author{
Lijalem Melie Tesfaw ${ }^{\oplus}$, Muluwerk Ayele Derebe ${ }^{\oplus}$, Haile Mekonnen Fenta ${ }^{\oplus}$ \\ Department of Statistics, Bahir Dar University, Bahir Dar, Ethiopia.
}

\begin{abstract}
Background. Lower Respiratory Tract Infections including pneumonia are the fourth cause of death globally. In Ethiopia, pneumonia is the leading cause of death for children under five. However, so far, only a few studies that used longitudinal design and time dependent covariates determined the significant factors of pneumonia. This study sought to determine whether respiratory rate changes differed for under five-year-old pneumonia patients who had been receiving different treatments over time and whether the change was effected by time dependent and independent covariates.
\end{abstract}

Methods. A longitudinal study design involving marginal Poisson regression models and conditional Poisson regression models was used. After comparing the two, the final interpretation was made using a conditional Poisson regression model owing to its relative powerfulness.

Results. Four hundred and fifty-three under five pneumonia patients were included, of which $44.37 \%$ were female. It is found that, compared to rural children, urban children had an estimated mean respiratory rate decrease of 3\%. It is also found that, compared to children whose mother practiced only exclusive breastfeeding in the first six months, children whose mother practiced both breastfed and complementary were more likely to be exposed to pneumonia. The estimated mean respiratory rate of children having asthma was 1.073 times that of children who had diarrhea.

Conclusions. In northwest Ethiopia, weight, residence, previous disease history, breastfeeding and temperature are significant factors of pneumonia among children under five. The effectiveness of treatments was dependent on the number of times children visited the hospital. A significant variation of baseline pneumonia status among under five pneumonia patient children was noted in the hospital.

Key words: pneumonia, respiratory rate, GEE, GLIMM, under five children.

Acute respiratory infection is an infection of the upper and lower respiratory system. Lower respiratory tract infections including pneumonia, affect the airways below the epiglottis specifically the lungs and are the cause of a high proportion of acute respiratory infection burden and are the fourth cause of death globally. ${ }^{1}$ Pneumonia is an acute illness of the lung alveolar air spaces that is

$凶 \quad$ Lijalem Melie Tesfaw

lijalemmelie@gmail.com

Received 15th May 2020, revised 16th December 2020, accepted 17th December 2020. diagnosed using respiratory rate (RR). Among patients hospitalized for respiratory system infections, children with pneumonia have a high proportion. According to 2019 UNICEF reports, compared to other infectious diseases, pneumonia which claims 2200 lives per day, nearly 800,000 per year, is the leading cause of mortality among children under five. ${ }^{1,2}$

Pneumonia, birth asphyxia, preterm birth complications and diarrheal diseases are the four major causes of mortality for under five children globally. Of these, Pneumonia ranked first cause of mortality and roughly accounts for $18 \%$ of the deaths among children under five. 
Malnourishment, AIDS, measles, malaria and environmental factors, such as crowded living conditions and exposure to indoor air pollution are more likely to contribute and an increased susceptibility to pneumonia in children. ${ }^{3}$ While possible causes of pneumonia are many, bacteria and viruses are among the more common. Though it is commonly diagnosed for the elderly (age $\geq 65$ years) and the very young (age $<5$ years), it is not potentially life threatening for adults. ${ }^{4}$

In developing countries in particular, of all deaths caused by acute respiratory infection among children aged under five years, pneumonia accounted for about $75 \%$ of the cases. ${ }^{2}$ A study conducted in East Africa reported that history of acute respiratory tract infection, wood as a fuel source, cooking food in the living room, being unvaccinated, exclusive breastfeeding, and prenatal smoking are independent important factors of pneumonia. 5,6 Nevertheless, although several precautions have been taken against pneumonia among under five children in Ethiopia, it remains the leading cause of death. ${ }^{7}$ A hospital-based prospective study found that the cut-off for breaths per minute was 50, 40 and 30 for infants (aged less than 12 months), children aged 1235 months and children aged 36-60 months, respectively. The study concluded the presence of high RR or fast breathing as an important indicator of pneumonia in all three age groups. ${ }^{2}$ Moreover, pneumonia of children under five was associated with low paternal education, the number of persons in the household and young maternal age. ${ }^{8}$

Compared to children who did not have a history of diarrhea, the odds of pneumonia were higher among children under five who had a history of diarrhea during the past fifteen days. ${ }^{9}$ In children with non-severe pneumonia in Pakistan, a placebo-controlled trial of amoxicillin showed insignificance benefit of amoxicillin over placebo. The study also noted a variation between children of Africa and Asia concerning etiology and the clinical course of pneumonia. This study further indicated that the body temperature of children at baseline was an important factor affecting their RR. ${ }^{10}$

UNICEF Ethiopia promoted a better and higher-quality diagnostic aid for pneumonia among young children through a public and private partnership. ${ }^{11}$ While immunization and breastfeeding are among the preventive, administration of amoxicillin tablets and other antibiotics were employed as curative methods. ${ }^{7}$ The study also indicated that female rather than male children were more likely to acquire pneumonia, indicating that the sex of children was an important factor. ${ }^{12}$ However, in a study of children 2 to 59 months old in the Arsi zone, Ethiopia, male children were more likely to develop pneumonia as compared to female children. ${ }^{13}$ Another study ${ }^{4}$, which diagnosed asthma and pulmonary TB indicated that, among adults, these did not have consistent significance on the community's acquisition rate of pneumonia. Compared to fully vaccinated children, unvaccinated children were more likely to develop pneumonia, and children whose parents practiced both breastfed and complementary fed during the previous six months were more likely to develop pneumonia as compared to children whose parents practiced exclusive breastfeeding. ${ }^{14,15}$

Several studies have demonstrated different factors that are significantly associated with pneumonia in children.,10,12,13 However, to the best of our knowledge, none of the studies considered time dependent factors but only time independent factors. Thus, this research paper aimed to build new insight on how to estimate the effect of time varying covariate including non-time varying covariates using an appropriate statistical model. Therefore, the objective of this study was to determine whether $R R$ changes differently for patients receiving different treatment over time and whether the change depends on time dependent and time independent patient characteristics. 


\section{Material and Methods}

Four hundred and fifty-three under five pneumonia patients were selected randomly from the Felege Hiwot Referral Hospital (FHRH) located in Bahir Dar, Amhara Region, Ethiopia, using stratified random sampling by considering their residence as strata. The data was collected from September 09 to 15, 2019. In FHRH as well as in Ethiopia, when a patient with pneumonia is hospitalized, registering the $\mathrm{RR}$ on the patients' registry card is mandatory. RR was measured by counting the respiratory chest movements, via inspection, and sometimes auscultation. So as to have reliable measured value of RR, a period of at least 30 seconds was allotted to account for the variability in measures of the breathing rate. In cases where the RR was difficult to detect within 30 seconds, data of RR over a period of 60 seconds or in two blocks of 30 seconds was used. ${ }^{2}$

The sample size was determined using single proportion formula with the following assumptions: estimated proportion of pneumonia patients $60 \%(=0.60), 95 \% \mathrm{CI}:\left(\mathrm{Z}_{\alpha / 2}\right.$ $=1.96)$, and a $5 \%$ margin of error $(d=0.05) \cdot{ }^{10,14}$ Hence, the final sample involved 453 pneumonia patients. Based on the recommendation of the physician in the hospital, patients under five with pneumonia were included. was determined as greater than 50 breaths per minute for those $<2$ months, 40 breaths per minute for those between 2-11 months, and greater than 30 breaths per minute for those between 1-5 years of age. ${ }^{2}$ The investigation didn't include those patients whose ages were greater than five years and children whose ages were under five years but had less than six visits in the study period.

\section{Variable description}

The outcome variable for this study was number of RR per minute in pneumonia patients under five years of age. The explanatory variables were categorized into two as: (i) time dependent covariates (TDC) such as treatment type (amoxicillin, ampicillin, ceftriaxone, gentamicin, penicillin), vomiting status (yes, no), temperature and time; (ii) time independent covariates (TIDC) such as sex (male, female), weight (in $\mathrm{kg}$ ), previous disease history (asthma, tuberculosis, malaria, diarrhea), immunization status (yes, no), weather (autumn, spring, summer, winter), breastfeeding (exclusive (B), complementary (C), and both breastfed and complementary fed and residence (urban, rural).

\section{Poisson Regression model (GEE approach)}

The generalized estimating equations (GEE) approach is a widely used estimation method for longitudinal marginal models. ${ }^{16,17}$ The within-subject correlations among the repeated measures are taken into account by using a working correlation structure and employing that structure for the parameter estimations. The statistical Poisson regression model is given by:

$\log (\mu i j)=\beta_{0}+\beta_{1} X_{i j 1}+\beta_{2} X_{i j 2} \ldots+\beta p X_{i j p}$

where, $\mu_{i j}$ is the expected RR of the $\mathrm{i}^{\text {th }}$ patient at the $j^{\text {th }}$ visit; $i=1,2, \ldots, 453$ and $j=1,2, \ldots, 6$; $\beta_{0}$ is the intercept while $\beta_{1^{\prime}}, \beta_{2^{\prime}}, \ldots, \beta_{p}$ are the coefficients of independent variables $X_{1}, X_{2}, \ldots, X_{p}$ respectively.

\section{Conditional Poisson Regression model}

GEE approach doesn't yield reliable results for a longitudinal model including random effects

and time-dependent covariates. Thus, generalized linear mixed model (GLMM) $)^{17,18}$ was applied to taking into under-consideration the random effects (random intercept and slops) and time dependent covariates, as given below by:

$\log (\mu i j)=\beta_{0}+\beta_{1} X_{1 i j}+\beta_{2} X_{2 i j}+\ldots+\beta_{p} X_{p i j}+\beta_{1}\left(t_{i j}\right) X_{1 i j}$
$+\beta_{2}\left(t_{i j}\right) X_{2 i j}+\ldots+\beta_{p}\left(t_{i j}\right) X_{p i j}+\alpha_{i}+b_{i} t_{i j}$ where $\beta_{1^{\prime}}, \beta_{2^{\prime}} \ldots, \beta_{p}$ are the only unknown coefficients of time independent covariates (TIDC) assumed to be constant while $\beta_{1}\left(t_{i j}\right)$, $\beta_{2}\left(t_{i j}\right), \ldots, \beta_{p}\left(t_{i j}\right)$ are unknown coefficient function of time that indicate coefficients of TDC change over time besides the corresponding constant 
coefficients $\beta_{1^{\prime}} \beta_{2}, \ldots, \beta_{p}$. Whereas, $\alpha_{i}$ and $b_{i}$ are random intercept and random slope of the $i^{\text {th }}$ child assumed to be independent and normally distributed with mean zero and constant variance.

Permission to undertake the study was obtained from both the ethical committees of Bahir Dar University and Felege Hiwot Referral Hospital (IRB number: 01-018/02.0). The researchers committed to the protection of the privacy of patients/participants medical information. Accordingly, identification of patients/ participants was done only through numerical codes and collection of medical information was made based on Ethiopian Ministry of health legislation to which FHRH is also committed. The children's parent gave written informed consent after having been introduced to the procedures, benefits and possible risks of participation in the study.

\section{Results}

\section{Exploratory data analysis}

Of the 453 pneumonia patients in the study, $44.3 \%$ were female, $77.26 \%$ were immunized/ vaccinated, and $50.55 \%$ were from a rural area (Table I). The majority of children (70.2\%) had no previous disease history while $13.91 \%$, $9.27 \%, 4.42 \%$ and $2.21 \%$ of the children had diarrhea, asthma, tuberculosis and malaria respectively. Most of the children (31.57\%) were born in the summer season. The mothers of $270(59.6 \%)$ children practiced both breastfed and complementary fedthe first six months of life. The proportion of children who received ceftriaxone, penicillin, gentamicin, amoxicillin and ampicillin treatment were $38.41 \%, 36.87 \%$, $10.15 \%, 7.51 \%$ and $7.06 \%$ respectively on their first visit (day 1). Similarly, 297 (65.56\%) children had persistent vomiting on their first visit (day 1).

For time dependent numerical variables, RR and temperature summary statistics were done for the first visit (day 1). On average, the temperature of under five pneumonia patients during their first visit was $37.8^{\circ} \mathrm{C}$ (Table II).

The individual profile plot in Figure 1 shows the evolution of RR of each child over time (six consecutive days), each line representing a child. The profile plot suggests that in nearly all of the children the RR declined with an increase in the time (in days) of a child's stay in the hospital. It also suggests the need for random intercepts to correct for the fact that the children started with different RR. Whereas, the slope for each profile plot of a child looks similar. The intermediate red line across the profile plots indicates the average estimated RR of a child which declined over time. We also indicated in Table III the average RR at day 1 was 58.67 and declined to 40.87 on day 6 .

Table I. Frequency distribution of under-five children within levels of categorical covariates.

\begin{tabular}{llc}
\hline Variable & Level & Frequency $(\%)$ \\
\hline Sex & Female & $201(44.37)$ \\
Weather & Male & $252(55.63)$ \\
& Autumn & $143(31.57)$ \\
& Summer & $119(26.27)$ \\
& Spring & $80(17.66)$ \\
Immunized & Winter & $111(24.5)$ \\
& No & $103(22.74)$ \\
Residence & Yes & $350(77.26)$ \\
& Rural & $229(50.55)$ \\
& Urban & $224(49.45)$ \\
& Asthma & $42(9.27)$ \\
Previous disease & Diarrhea & $63(13.91)$ \\
& No & $10(2.21)$ \\
& Tuberculosis & $318(70.2)$ \\
Breast feeding & $20(4.42)$ \\
& Exclusive (E) & $159(35.1)$ \\
& Complementary(C) & $24(5.3)$ \\
& Both breastfed and & $270(59.6)$ \\
& Complementary fed & \\
Vomiting status & No & $156(34.44)$ \\
& Yes & $297(65.56)$ \\
& Amoxicilline & $34(7.51)$ \\
& Ampicillin & $32(7.06)$ \\
& Ceftriaxone & $174(38.41)$ \\
& Gentamicin & $46(10.15)$ \\
& Penicillin & $167(36.87)$ \\
\hline \multirow{5}{*}{ Treatment } &
\end{tabular}


Table II. Summary statistics of numerical covariates of children under-five who visit FHRH.

\begin{tabular}{lccccc}
\hline Variable & $\mathrm{N}$ & Mean & St. Dev & Minimum & Maximum \\
\hline Respiratory rate & 453 & 58.67 & 9.24 & 32.00 & 84.00 \\
Temperature & 453 & 37.80 & 1.02 & 32.50 & 40.10 \\
Weight & 453 & 7.83 & 2.91 & 2.00 & 18.00 \\
\hline
\end{tabular}

Table III. Average and standard deviation (St.dev) of respiratory rate per day.

\begin{tabular}{lcccccc}
\hline \multirow{2}{*}{ Statistic } & \multicolumn{7}{c}{ Time (in days) } \\
\cline { 2 - 7 } & Day1 & Day2 & Day3 & Day4 & Day5 & Day6 \\
\hline Average & 58.67 & 54.72 & 50.67 & 47.19 & 43.97 & 40.87 \\
St.dev & 9.24 & 8.97 & 8.30 & 7.97 & 7.39 & 6.87 \\
\hline
\end{tabular}

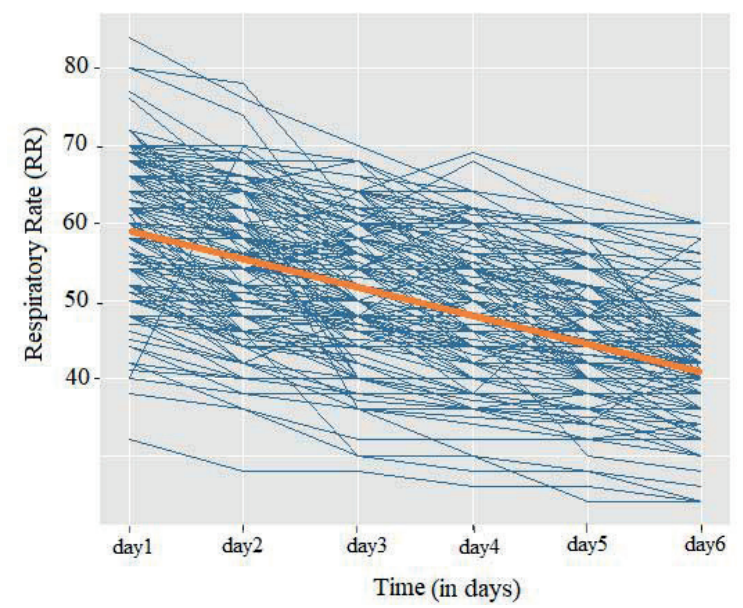

Fig. 1. Individual profile plot of respiratory rate of children over time (in days).
In addition to trends of average $R R$, the variability of RR declined over time (9.24 on day 1 to 6.87 on day 6) indicating that the RR of children in the hospital becomes more consistent as the number of days of staying in the hospital increased.

Figure 2 revealed the progress of the average RR of children over six consecutive days per treatment type. The average RR of children per each treatment type declined (supported from Fig. 1) and became close to similar. The average RR of children treated with Amoxicillin was the lowest throughout all of the days.

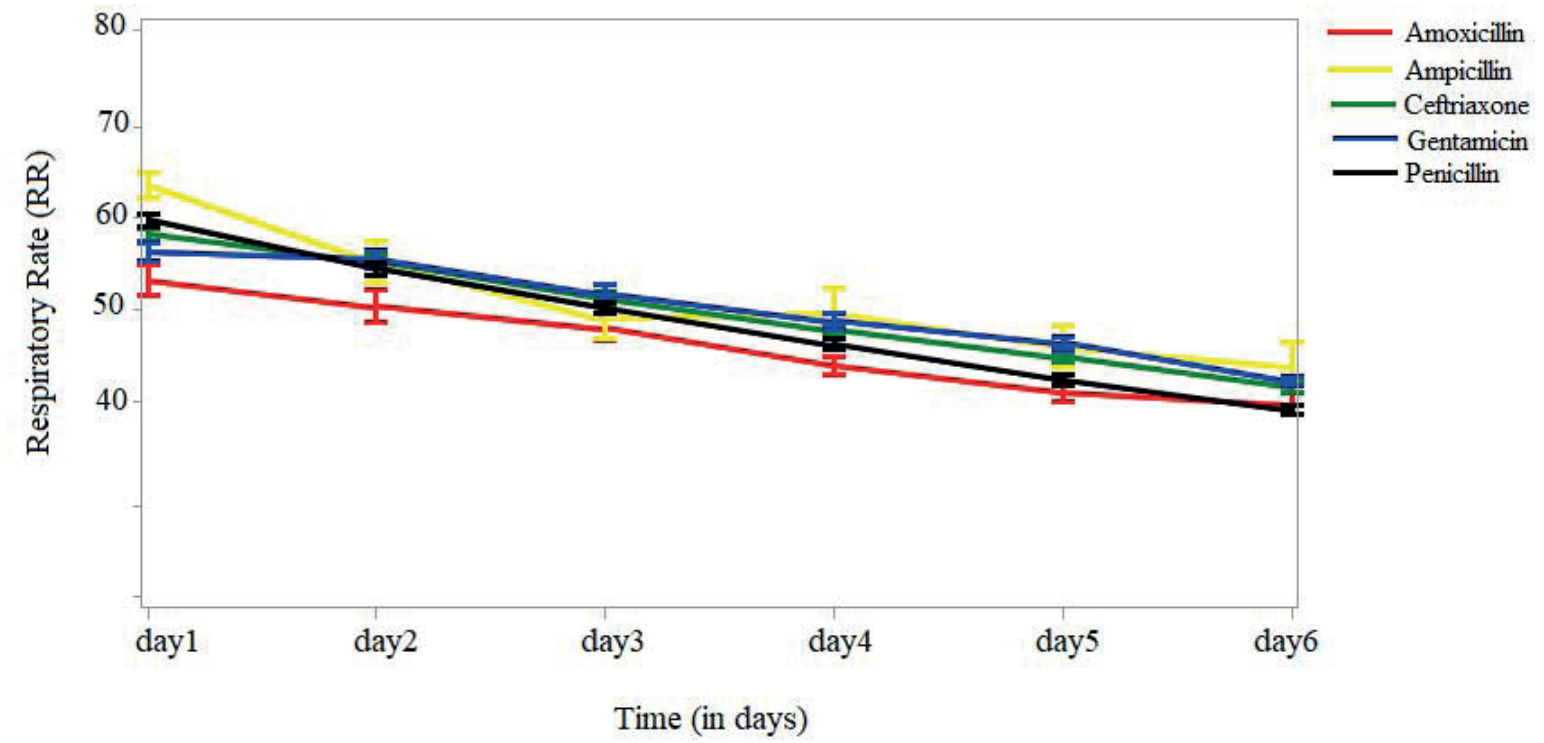

Fig. 2. Average respiratory rate of children per each treatment type represented as Amoxicillin, Ampicillin, Ceftriaxone, Gentamicin and Penicillin with red, yellow, green, blue and black color line respectively. 


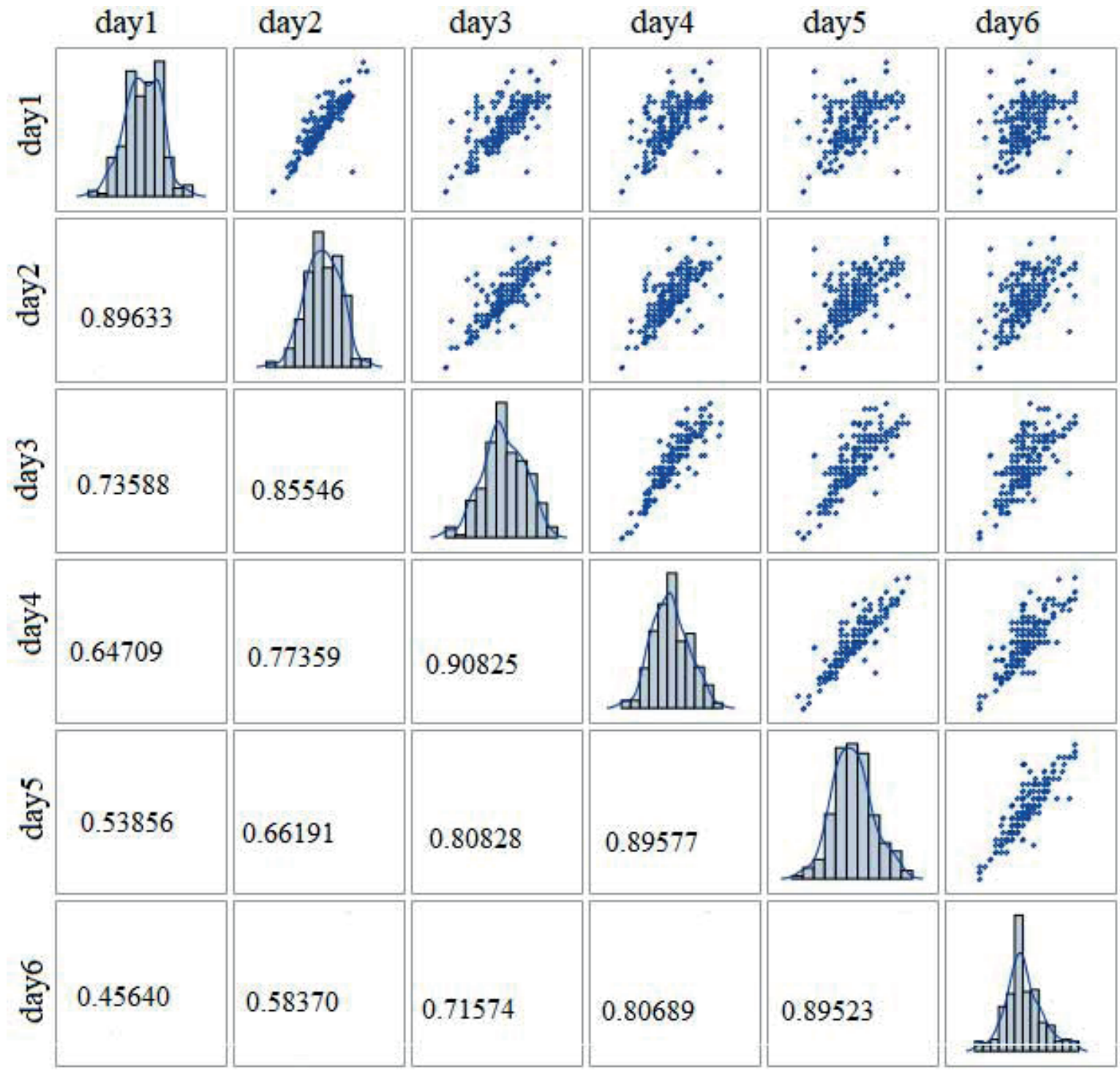

Fig. 3. Scatter correlation matrix.

The scatter matrix plot in Figure 3 indicates a positive correlation between RR on different days as there is a positive slope. The correlation was presented in form of a scatter plot (see the diagonal matrix in Fig. 3) and corresponding numerical expression (see the diagonal matrix in Fig. 3). The correlation between RR decreased with an increase in time (in days). The decrease in correlation overtime has an implication of autoregressive working correlation ${ }^{19}$, decline in the correlations with increasing separation in time.

\section{Parameter estimates}

The parameter estimates for the covariates in the study are presented in Table IV and V. Table IV presents the Poisson regression model obtained using GEE which was compared according to different working correlations such as independent GEE (IGEE), compound symmetry GEE (CSGEE) and autoregressive GEE (ARGEE). The comparison was made using QIC, and the minimum the QIC, the better the model. ${ }^{16}$ In this regard, a Poisson regression model using ARGEE is the best model, with QIC $=440$. In the standard Poisson regression model that 


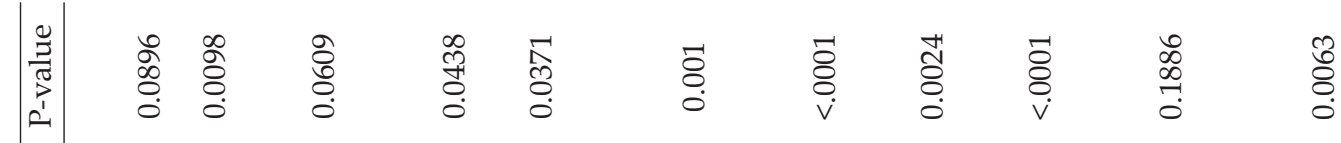

弥

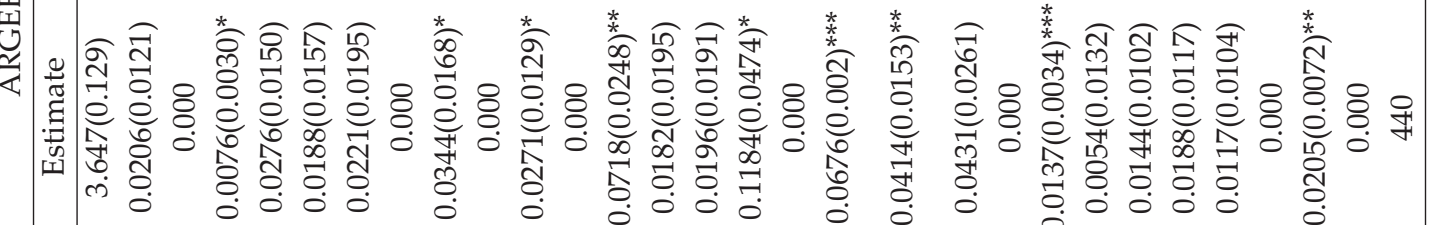

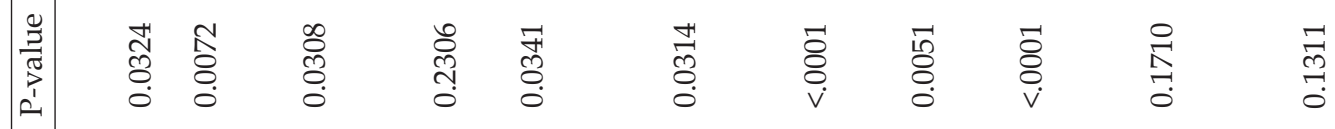

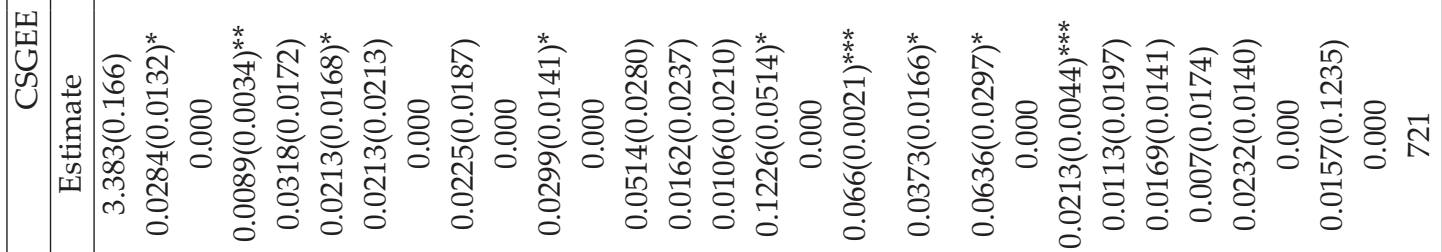

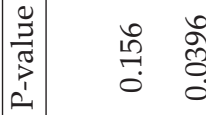

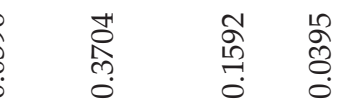

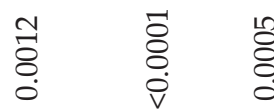

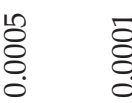
$\begin{array}{lll}0 & \infty & 0 \\ 0 & 0 & 0 \\ 0 & 0 & 0 \\ 0 & 0 & 0\end{array}$

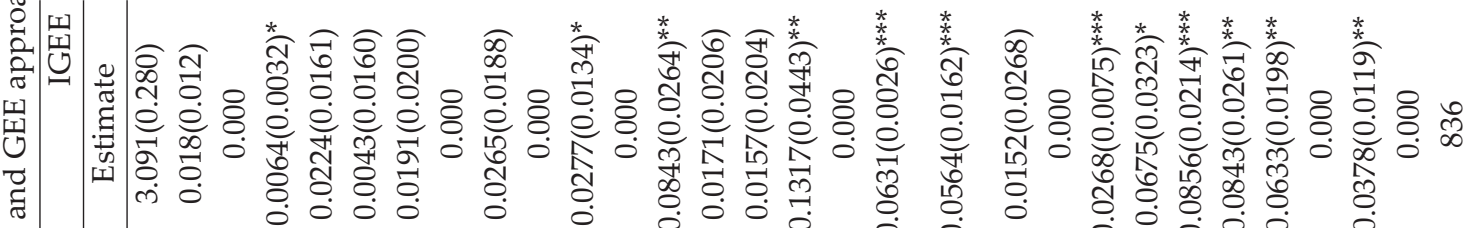

$\stackrel{8}{0}$

$\stackrel{10}{2}$

$\stackrel{m}{8} \stackrel{8}{8}$

$\begin{array}{ll}\vec{\delta} & \overline{8} \\ \dot{8} & \dot{8}\end{array}$

客

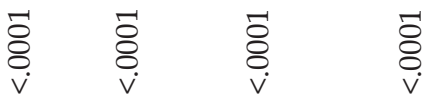

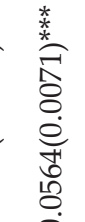

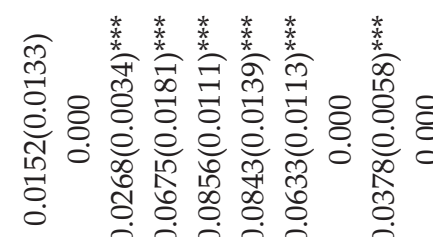

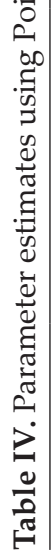

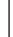

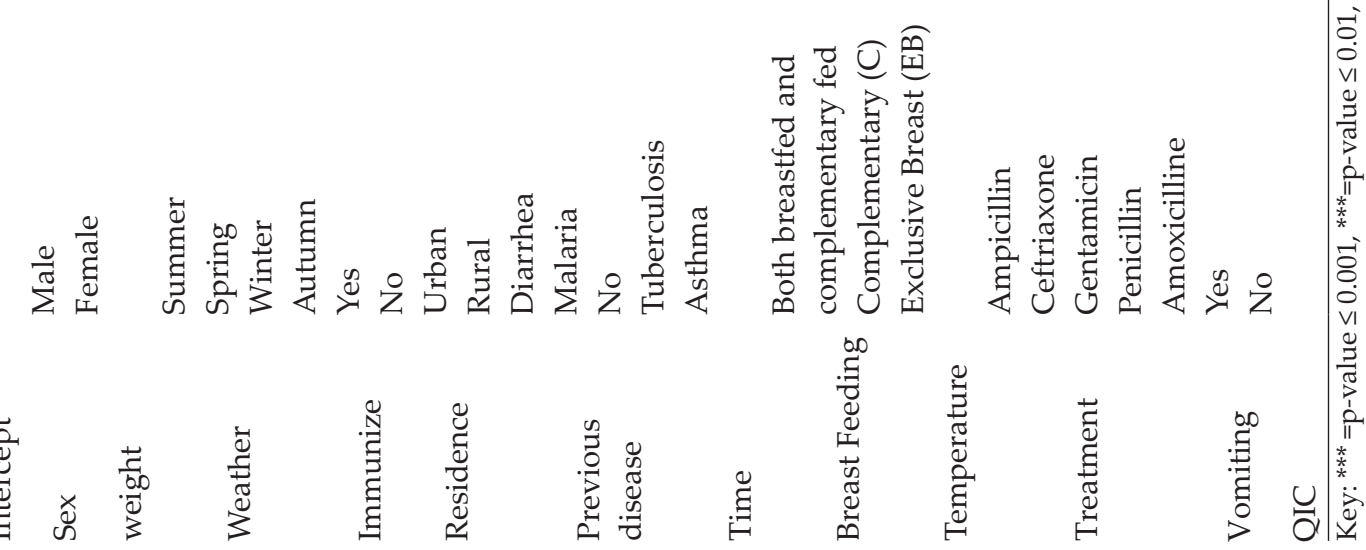




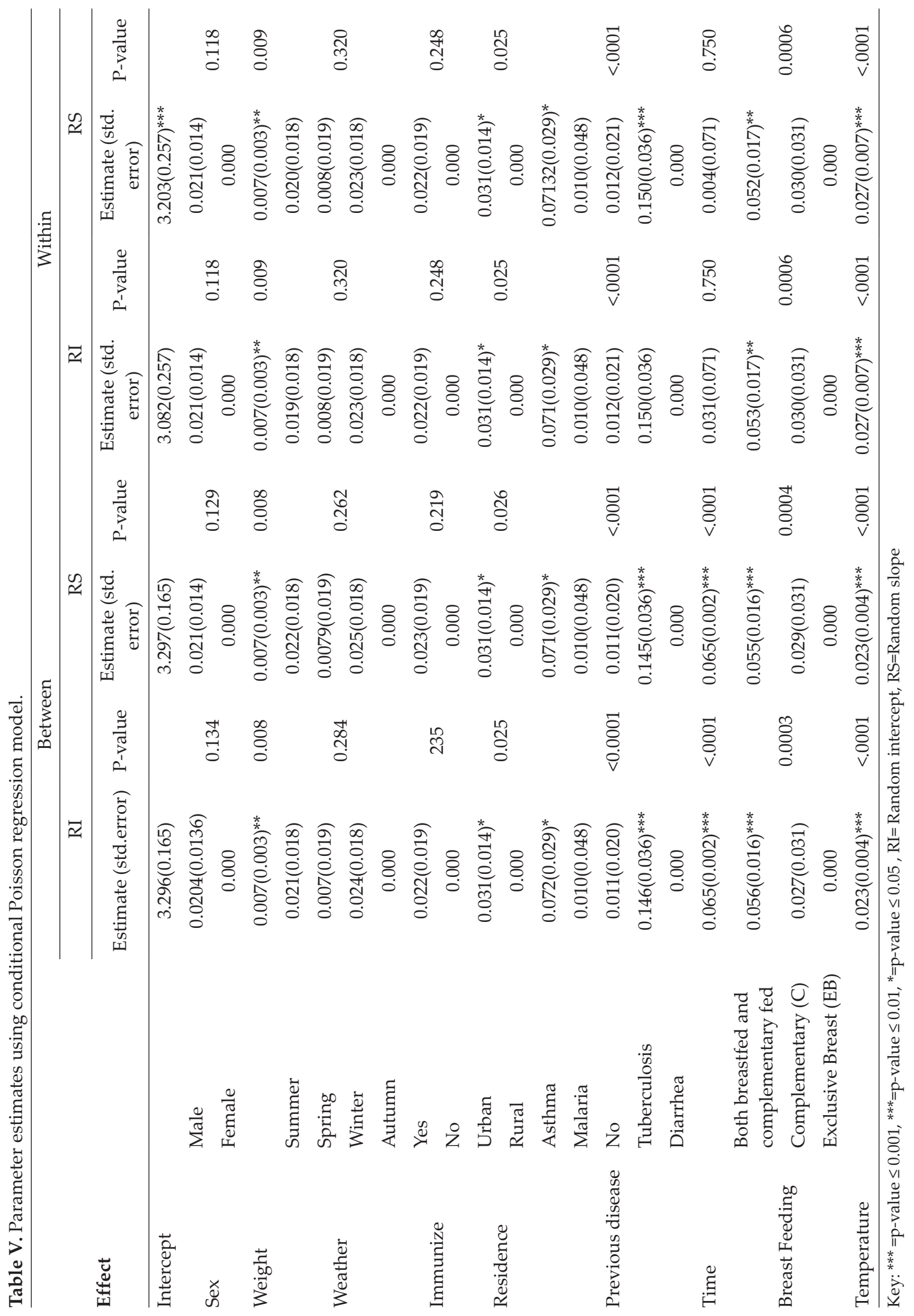




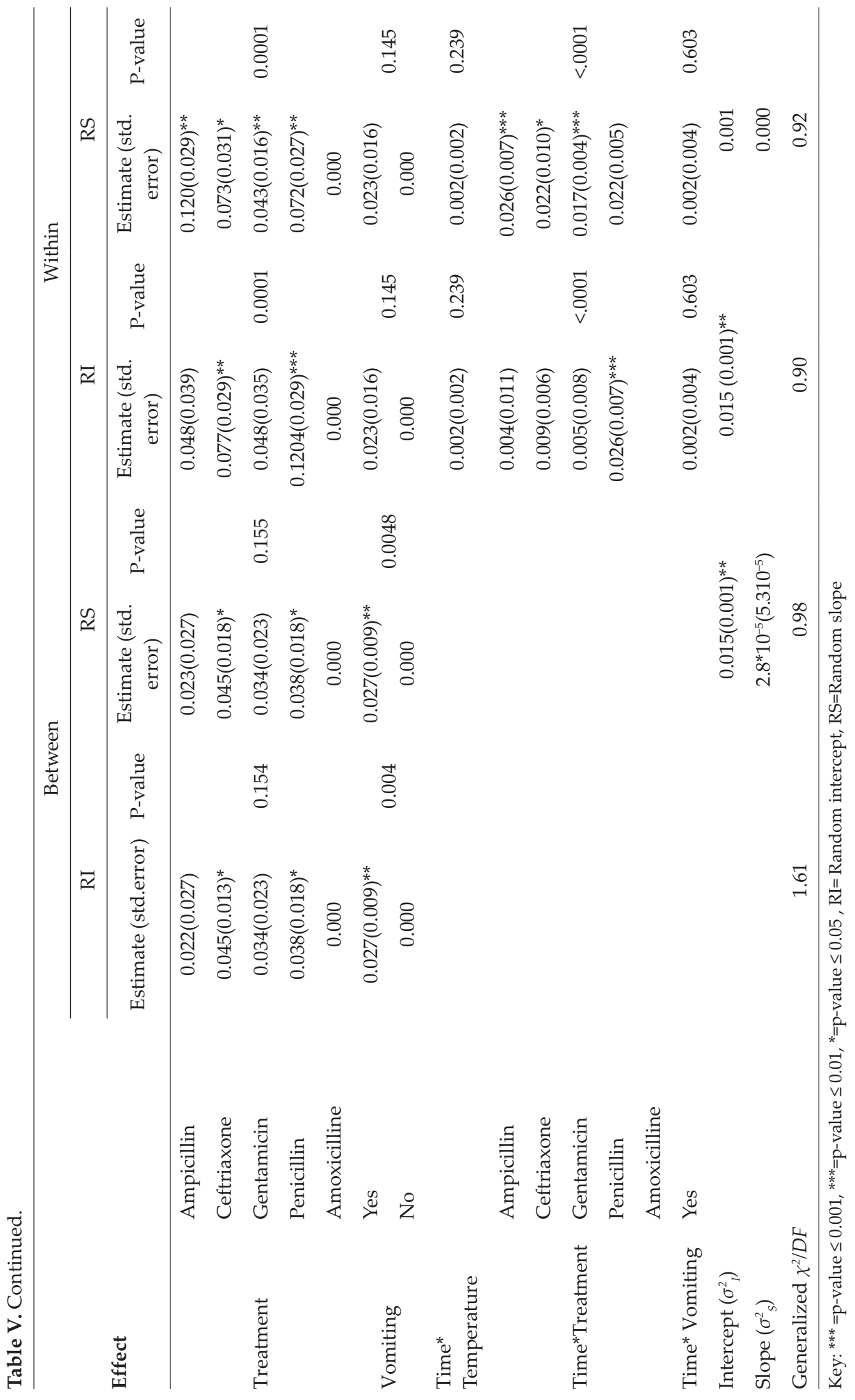


assumes each observation is independent, all the variables included in the model had a significant association ( $\mathrm{p}$-value $<0.05$ ) with RR. However, when the correlation between measurements is considered, the number of variables having a significant association with RR decreased. This indicates that considering the correlation measurement within each child is necessary. However, as considering the correlation only may not be effective, identifying the working correlation is also important.

As we observed from the scatter matrix plot in Figure 3 and checked with the minimum QIC, the best working correlation of the marginal model is ARGEE. Characteristics of children such as weight, immunization, residence, previous disease history, number of hospital visits, breastfeeding, body temperature and vomiting status have a significant effect ( $p$-value $<0.05$ ) on $R R$. The estimated mean RR count decreased by $1 \%\left(\mathrm{e}^{-0.0076}=0.99\right)$ for a unit weight increase by keeping other characteristics constant. The estimated mean RR count of children who were living in an urban area decreased by $3 \%$ $\left(\mathrm{e}^{-0.0271}=0.97\right)$ meaning that children who were born in an urban area were less likely to have pneumonia. When the number of visits of a child to the hospital increased by one, the estimate RR decreased by $7 \%\left(\mathrm{e}^{-0.0676}=0.93\right)$.

Moreover, compared to children whose mothers practiced both breastfed and complementary fed, children whose mothers practiced exclusive breastfeeding had greater decrement RR $\left(\mathrm{e}^{0.0414}=1.042\right)$. The estimated mean RR increased by $1.4 \%\left(\mathrm{e}^{0.0137}=1.014\right)$ for a unit increase in temperature. The estimated mean $R R$ of children who were currently vomiting was $1.021\left(\mathrm{e}^{0.0205}\right)$ times of the children who weren't vomiting. This indicates that the RR of vomiting children were greater by $2.1 \%$.

The model includes determining the random effects (random intercept and random slope) within decomposition (within and between) of the time-dependent covariates (Table V). The conditional Poisson regression model was fitted by including a decomposition into "within" and "between" components of time-dependent covariates in Table V. In each of "within" and "between" time dependent covariates decomposition of the random intercept (RI) and random slope (RS) was presented. Once the parameters are estimated, the conditional Poisson regression model comparison was made using generalized $\chi^{2} / D F$ as a fit statistic that incorporate over dispersion. The model with lower generalized $\chi^{2} / D F$ and parsimony is the better ${ }^{15}$ and hence, a random intercept Poisson regression model $\left(\chi^{2} / D F=0.90\right)$ that considers the dynamic relationship of time dependent covariates such as treatment, temperature and vomiting status is preferred. The model illustrates that the baseline RR of children differ (random intercept, $\sigma^{2}=0.015$ ) while the progress of RR (random slope) of children over time didn't, which matches with the profile plot in Figure 1.

Demographic characteristics of the children such as weight, residence, previous disease history, breastfeeding and temperature have significant effects on the RR. Similarly, the dynamic effect of treatment over time (treatment*time) also has a significant effect (p-value $<0.05$ ). The estimated mean RR of pneumonia patients decreased by $1 \%\left(e^{-0.007}=0.99\right)$ per a unit increase of weight (in $\mathrm{Kg}$ ). Besides, compared to the estimated mean RR of children from rural areas, urban children have an estimated mean RR decreased by $3 \%$ $\left(e^{-0.031}=0.97\right)$. This indicates that children from urban areas were more likely to have a lower $R R$, and were less vulnerable to pneumonia.

Compared to the estimated mean RR of both breastfed and complementary fed children, the estimated mean RR of children receiving exclusive breastfeeding, was higher by $5 \%$ $\left(e^{0.053}=1.054\right)$. This indicating that compared to mothers who practice both breastfed and complementary fed during the first six months, mothers who practiced only exclusive breastfeeding reduced the susceptibility of their child from pneumonia. On the other hand, for a unit increase of temperature, the estimated mean RR increased by $2.7 \%\left(e^{0.027}=\right.$ 1.027). There was also a significant estimated 
mean RR difference between children with and without previous diseases history of malaria, tuberculosis, asthma, and diarrhea. For instance, the estimated mean RR of children with asthma was $1.073\left(e^{0.071}\right)$ times that of the estimated mean RR of children with diarrhea.

\section{Discussion}

The main aim of this study was to describe the evolution of RR of pneumonia in children under five and to determine the effect of treatment, and time dependent and independent characteristics on RR. Two statistical model approaches were used: marginal Poisson regression model (standard and GEE approach) and conditional Poisson regression model (random intercept and slope with the decomposition of time dependent covariates). Model comparison was carried out and parameters estimated using the better of the two models. Both approaches ended up with similar results except that the conditional Poisson regression model analysis added up another information about the baseline RR difference and dynamic relationship of time dependent covariates. This indicates that parameter estimates that used the conditional Poisson regression model was more powerful to meet our objectives.

In general, for the six visits (day 1 to day 6) a decrease of RR counts was observed, this indicates that an increase in the number of days visiting the hospital from (day1 to day 6) correlated with a decrease in the RR. The baseline RR had significant variability which is consistent with a previous study ${ }^{10}$, in which both infants and older children showed a random variation in $R R$ over relatively short periods of time or inaccuracies in measurement. The decrease in the RR of each child was nearly similar. The average RR of children in each treatment type declined and was close to each other for each treatment. Ceftriaxone was the most commonly prescribed treatment $(38.41 \%)$ while Ampicillin treatment was the least prescribed $(7.06 \%)$. The distribution of urban and rural patients was nearly equal $(50.55 \%$, and $49.45 \%$ respectively). It was noted that most children $(13.91 \%)$ had a previous disease history of diarrhea. Of the participants $65.56 \%$ had vomiting.

The place of residence of the children (rural or urban) and previous disease history had a significant effect on their RR. These findings are in line with a study that reported that prior disease history such as malaria and environmental factors, such as crowded living conditions and exposure to indoor air pollution may contribute to increase susceptibility to pneumonia. $^{3}$ A study on children between 2 to 59 months in Arsi zone, Ethiopia, reported that among factors associated with pneumonia, compared to female children, male children were more likely to develop pneumonia. ${ }^{13}$ This study, however, didn't find a significant RR difference in male and female children. Compared to children who had no previous disease history, children with tuberculosis and diarrhea had an increased susceptibility to pneumonia. However, in another study it was reported that among adults with community acquired pneumonia the diagnosis of asthma and pulmonary TB didn't have a consistent significance. ${ }^{4}$ Compared to children whose mother practiced only exclusive breastfeeding, children whose mother practiced both breastfed and complementary fed were more likely to catch pneumonia especially in children who are in the first six months of age. ${ }^{14}$ Baseline body temperature of children during diagnosis had a significant effect on the RR of under five children, that comply with the finding of a study by Muro et $\mathrm{al}^{10}$, which reported the higher the temperature of the children, the higher their RR. The effect of treatment on $R R$ varied within time in the sense that treatment effectiveness differed according to the number of times the children visited the hospital. Finally, as the weight of the children decreased, they were more likely to have a higher RR indicating that the weight of under five children has a significant effect on their RR. Therefore, this study concludes that weight, residence, previous disease history, breastfeeding and temperature were found 
to be significant factors of pneumonia among children under five in northwest Ethiopia. The effectiveness of treatments co-varied with the number of times the children visited the hospital. A significant variation of baseline pneumonia status was also noted among the children.

\section{Acknowledgment}

The authors are thank full to personnel and experts in FHRH for the data availability and expertise assistance.

\section{Author contribution}

The authors confirm contribution to the paper as follows: study conception and design: HMF, MAD, LMT; analysis and interpretation of results: LMT; draft manuscript preparation: LMT. All authors reviewed the results and approved the final version of the manuscript.

\section{Ethical approval}

Permission to undertake the study was obtained from both the ethical committees of Bahir Dar University and Felege Hiwot Referral Hospital (IRB number: 01-018/02.0).

\section{Availability of data and material}

The datasets for the generated analyses during the study is freely available in FHRH, Bahir Dar, Ethiopia by offering formal request of the research proposal.

\section{Source of funding}

No funding was obtained for this study

\section{Conflicts of interests}

The authors declare that they have no competing interests.

\section{REFERENCES}

1. Unicef's report of pneumonia 2019: 1-5. Available at: https://data.unicef.org/topic/child-health/ pneumonia/ (Accessed on January28, 2020).

2. Gupta D, Mishra S, Chaturvedi P. Fast breathing in the diagnosis of pneumonia-a reassessment. J Trop Pediatr 1996; 42: 196-199.

3. Tong N. Priority Medicined for Europe and the World. A Public Health Approach to Innovation. Background Paper 6.22 Pneumonia, 2013. Available at: https://www.who.int/medicines/areas/priority_ medicines/BP6_22Pneumo.pdf (Accessed on May 16, 2021).

4. Gebru HB, Gahse FE, Kahsay AB. Risk factors of community acquired pneumonia among adults in Tigray, Ethiopia: a case-control study. J Clin Diagn Res 2018; 12: LG01-LG05.

5. Beletew B, Bimerew M, Mengesha A, Wudu M, Azmeraw M. Prevalence of pneumonia and its associated factors among under five children in East Africa: a systematic review and meta-analysis. BMC Pediatr 2020; 20: 254

6. Karki S, Fitzpatrick AL,Shrestha S. Risk factors for pneumonia in children under 5 years in a teaching hospital in Nepal. Kathmandu Univ Med J 2014; 12: 247-252.

7. UNICEF reports of pneumonia in Ethiopia 2014: 2-10. https://unicefethiopia.org/2014/11/13/ inethiopia-pneumonia-is-a-leading-single-diseasekilling-under five-children/ (Accessed on December 28, 2019).

8. Victora CG, Fuchs SC, Flores JA, Fonseca W, Kirkwood B. Risk factors for pneumonia among children in a Brazilian metropolitan area. Pediatrics 1994; 93(6 Pt 1):977-985.

9. Markos Y, Dadi AF, Demisse AG, Ayanaw Habitu Y, Derseh BT, Debalkie G. Determinants of under five pneumonia at Gondar University Hospital, Northwest Ethiopia: an unmatched case-control study. J Environ Public Health 2019; 2019: 9790216.

10. Muro F, Mtove G, Mosha N, et al. Effect of context on respiratory rate measurement in identifying nonsevere pneumonia in African children. Trop Med Int Health 2015; 20: 757-765.

11. UNICEF annual report of pneumonia in Ethiopia 2017: 1-5. https://www.unicef.org/ about/ annualreport/files/Ethiopia 2017 COAR.pdf (Accessed on January, 2020). 
12. Negash AA, Asrat D, Abebe W, et al. Bacteremic community-acquired pneumonia in Ethiopian children: etiology, antibiotic resistance, risk factors, and clinical outcome. Open Forum Infect Dis 2019; 6: ofz029.

13. Lema B, Seyoum K, Atlaw D. Prevalence of community acquired pneumonia among children 2 to 59 months old and its associated factors in Munesa district, Arsi Zone, Oromia Region, Ethiopia. Clin Mother Child Health 2019; 16: 334.

14. Lema KT, Murugan R, Tachbele E, Negussie BB. Prevalence and associated factors of pneumonia among under five children at public hospitals in Jimma zone, South West of Ethiopia 2018. J Pulmonol Clin Res 2018; 2: 25-31.

15. Strauß R, Ewig S, Richter K, König T, Heller G, Bauer TT. The prognostic significance of respiratory rate in patients with pneumonia: a retrospective analysis of data from 705,928 hospitalized patients in Germany from 2010-2012. Dtsch Arztebl Int 2014; 111: 503-508.
16. Molenberghs G, Verbeke G. Models for Discrete Longitudinal Data (Springer Series in Statistics). (Kindle ed) New York: Springer Science and Business Media, 2005.

17. Lalonde TL, Nguyen AQ, Yin J, Irimata K, Wilson JR. Modeling correlated binary outcomes with timedependent covariates. Data Sci J 2013; 11: 715-738.

18. Cai K, Wilson JRHow to use SAS ${ }^{\circledR}$ for GMM logistic regression models for longitudinal data with timedependent covariates. InProceedings of the SAS Global Forum Conference 2015: 1-8.

19. Guerra MW, Shults J, Amsterdam J, Ten-Have T. The analysis of binary longitudinal data with timedependent covariates. Stat Med 2012; 31: 931-948. 